\title{
Muertes maternas en el Hospital universitario san Vicente de Paul
}

DR. ALFONSO JUBIZ HASBUN*

DRA. CARMEN SANCHEZ MALDONADO**

\section{INTRODUCCION}

El estudio de las muertes maternas nos indica con bastante exactitud el tipo de atención que se presta a las embarazadas en cualquier certro asistencial; a la vez, mide el desariolio de un país. Las madres y los niños menores de 5 años representan las dos terceras partes $(2 / 3)$ de la población mundial, es lógico pensar entonces que la calidad de la atención maternoinfantil debe ser prioritaria en cualquier programa de atención médica.(1).

Los cuidados de la madre y de su hijo deben encaminarse hacia una educación adecuada, una supervisión de la salud, unos cuidados médicos en general y un control prenatal en particular.

Durante el embarazo es muy importante cumplir unos objetivos que nos permitan brindar a la embarazadia una supervisión de su salud y proporcionarle tratamiento correcto para sus problemas, muchos de ellos sin ninguna relación con el embarazo, pero que pueden ser en última instancia la causa de là muerte. Además, desde el comienzo de la gestación y durante el progreso de la misma, se deben adecuar los recursos para asegurarle al recién nacido unos cuidados que le permitan un desarrollo normal.

Los adelantos alcarizados en la obstetricia y en lia anestesia han permito disminuir las cifras de la mortalidad materna, sin embargo en muchos países, incluyendo el nuestro, el $75 \%$ de las muertes se producen por la infección, la toxemia y la hemorragia y es más desalentador el estudio de estas muertes cuando casi el $90 \%$ de ellas se pueden prevenir. (2).

Es difícil muchas veces, indicar el grado de responsabilidad del personal médico, pa-

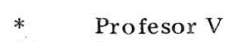

** Instructora

Depto. de Obstetricia y Ginecología ramédico, del centro asistencial ò de la paciente. Debemos luchar entonces para que nuestros errores sean cada ve\% menores y para que la atención materno infantil alcance cada día el primer lugar en los programas del Ministerio de Salud Pública.

\section{OBJETIVO:}

Analizar las causas directas, indirectas y no relacionadas de las muertes maternas ocurridas en el Departamento de Obstetricia y Ginecología (Universidad de Artioquia - Hospital Universitario San Vicente de Paúl) desde 1963 hasta 1976.

\section{MATERIAL Y METODOS:}

Se revisaron las 149 historias cie muertes maternas ocurridas en el Departamento de Obstetricia y Ginecología (Hospital Universitario San Vicente de Paúl) desde 1963 hasta 1976 inclusive y se analizaron las siguierates variables:

3.1. Elad de la Paciente

3.2. Procedencia

3. 3 Estado Civil

3.4. Semanas de gestación

3.5. Paridad

3.6. Número de consultas prenatales

3.7. Terminación del embarazo

3.8. Tipo de aborto

3.9. Antecedentes de maniobras abortivas

Para las causas de muerte tuvimos en cuenta la clasificación recomendada por la Asociación Médica Americana (3).

Aceptamos como muerte materna aquella que ocurre por cualquier causa durante el embarazo, parto y los 90 días siguientes a la terminación del embarazo, independiente de la duración y del sitio del mismo, por 10.000 nacidos vivos (3).

A. Muerte por causa directa: Es aquella que resulta de cualquier complicación del em- 
barazo, parto o puerperio y que fue resultado de una serie de hechos que se iniciaron por una complicación o una intervención. B. Muerte por causa indirecta: La muerte es debida a una enfermedad desarrollada antes o durante el embarazo, la cual fue agravada por los efectos fisiológicos del mismo, pero no por causa directa del embarazo.

C. Muerte por causa no relacionada: Cuando la muerte ocurre durante el embarazo, parto o puerperio por causas no relacionadas con el embarazo, ni con sus complicaciones o manejo.

Durante los 14 años revisados se atendieron 60.057 partos y el número de nacidos vivos fue de 58.700 .

La edad gestacional se calculó en semanas según la fecha de la última menstruación consignada en la historia.

La variable paridad incluye solamente los partos o cesáreas de las gestaciones a término.

El estudio anatomopatológico completo (necropsia), se realizó en 62 de las pacientes fallecidas. Para determinar la causa de la muerte cuando no se encontró este estudio, nos basamos en los datos histológicos de algunas piezas quirúrgicas y además sè hizo un análisis detenido y una discusión de cada historia.

\section{RESULTADOS:}

4.1 Edad de la Paciente: (Cuadro No. 1)

\begin{tabular}{|c|c|c|}
\hline \multicolumn{3}{|c|}{ EDAD } \\
\hline AÑOS & Númer & $\%$ \\
\hline $15-24$ & 34 & 22.8 \\
\hline $25-34$ & 64 & 42.9 \\
\hline $35-44$ & 49 & 32.9 \\
\hline 45 o más & 2 & 1.4 \\
\hline TOTAL & 149 & 100.0 \\
\hline
\end{tabular}

Las edades estuvieron comprendidas entre 15 y 47 años y prácticamente la totalidad de las pacientes fallecidas se encontraban en el período normal de reproducción.

4.2 Procedencia:
Al analizar esta variable llama la atención el hecho de que el $55.7 \%$ (83 pacientes) procedían del área urbana de la ciudad de Medellín, donde existen adecuados recursos obstétricos.

El $38 \%$ consultaron al hospital remitidas del área rural.

\subsection{Estado Civil:}

El $70 \%$ de las madres fallecidas eran casadas y esto es el reflejo de lo que ocurre con la gran mayoría de las pacientes que ingresan a nuestro departamento.

4.4 Semanas de Gestación:

Es importante resaltar que el $36.2 \%$ de las pacientes tenían una edad gestacional inferior a 28 semanas, el $15.4 \%$ un embarazo a término. El $20.8 \%$ ingresaron con un embarazo entre 28 y 38 semanas y un porcentaje igual en la etapa del posrparto.

\subsection{Paridad: (Cuadro No. 2)}

\begin{tabular}{|l|r|r|}
\hline \multicolumn{3}{|c|}{ PARIDAD } \\
\hline No. DE HIJOS & No. DE PTES. \\
\hline Primigestantes & 14 & 9.4 \\
\hline $1-3$ & 56 & 37.6 \\
\hline $4-6$ & 26 & 17.4 \\
\hline 7 o más & 45 & 30.2 \\
\hline Desconocido & 8 & 5.4 \\
\hline TOTAL & 149 & 100.0 \\
\hline
\end{tabular}

El grupo de primigestantes fue solo de 9.39\%. La frecuencia mayor recayó en las madres con 1 a 3 hijos (37.6\%), seguido por el de las grandes multíparas (30.2\%).

\subsection{Número de consultas prenatales:}

Por diferentes motivos que son muy importantes y que se deben averiguar, en nuestro medio las embarazadas continúan alejadas de la consulta prenatal. Solo el $11.4 \%$ tuvieron esa atención durante la gestación. Si se tiene en cuenta que la mayoría de las veces es tardía y escasa, se podría pensar que este factor está jugando un papel importante en las causas de las muertes 
maternas en nuestro medio

4.7 Terminación del embarazo (Cuadro No. 3).

\begin{tabular}{|l|c|c|}
\hline \multicolumn{3}{|c|}{ RESULTADO FINAL DEL EMBARAZO } \\
\hline ESTADO & Número & $\%$ \\
\hline Vivos & 47 & 31.5 \\
\hline Muertos & 41 & 27.5 \\
\hline Abortos & 54 & 36.2 \\
\hline Desconocido & 7 & 4.8 \\
\hline TOTAL & 149 & 100.0 \\
\hline
\end{tabular}

Prácticamente en el $70 \%$ de los embarazos de las madres fallecidas el producto de la concepción se perdió en etapa precoz, intermedia o tardía y sólo en el $31.5 \%$ el recién nacido sobrevivió a la madre.

\subsection{Tipo de Aborto:}

El $70.3 \%$ de los abortos fueron infectados, sin embargo, solo en 4 casos, se encontró que habían sido provados. El $33.3 \%$ de los abortos tenían una edad gestacional entre 5 y 12 semanas.

4.9 Causas globales de muerte: (Cuadro 4)

\begin{tabular}{|c|c|c|}
\hline \multicolumn{3}{|c|}{ CAUSAS DE MUERTE. } \\
\hline CAUSAS & Número & $\%$ \\
\hline Infección & 66 & 44.3 \\
\hline Indirectas & 32 & 21.4 \\
\hline Toxemia & 30 & 20.1 \\
\hline Hemorragia & 21 & 14.2 \\
\hline TOTAL & 149 & 100.0 \\
\hline
\end{tabular}

La infección ocupó el primer lugar como causa de muerte con $44.3 \%$, seguida por las causas indirectas con $21.4 \%$ entre éstas merecen destacarse 3 paros cardíacos durante la anestesia y I infarto del miocardio. El tercer lugar le correspondió a la toxemia con $20.1 \%$ y finalmente la hemorragia con $14.2 \%$.

\subsection{Hemorragia: (Cuadro No. 5)}

\begin{tabular}{|l|c|c|}
\hline \multicolumn{3}{|c|}{ HEMORRAGIA } \\
\hline CAUSAS & Número & $\%$ \\
\hline Desp. prem. de & & \\
\hline placenta & 7 & 33.3 \\
\hline Ruptura uterina & 6 & 28.6 \\
\hline Acretismo placentario & 5 & 23.8 \\
\hline Placenta previa & 3 & 13.6 \\
\hline
\end{tabular}

El desprendimiento prematuro de la placenta fue la principal causa de muerte por hemorragia con $33.3 \%$, en segundo lugar estuvo la ruptura uterina con $28.6 \%$, luego la placenta acreta con 5 casos $(23,8 \%)$ y por último la placenta previa con $14.3 \%$

\subsection{Infección: (Cuadro No. 6)}

\begin{tabular}{|l|c|c|}
\hline \multicolumn{3}{|c|}{ INFECCION } \\
\hline & Número & \multicolumn{1}{|c|}{} \\
\hline Aborto & 38 & 57.0 \\
\hline Parto y puerperio & 28 & 43.0 \\
\hline TOTAL & 66 & 100.0 \\
\hline
\end{tabular}

De las 66 embarazadas muertas por infección, el $57 \%$ derivaron esta complicación de un aborto y el $43 \%$ por un proceso séptico del parto o del puerperio.

\subsection{Toxemia:}

El $76.6 \%$ de las pacientes fallecidas por esta causa tenían una pre-eclampsia grave y las restantes una eclampsia.

\section{COMENTARIOS:}

En un trabajo anterior (4), comentamos cómo el principal centro asistencial del departamento de Antioquia se ha convertido en un hospital de Alto Riesgo, no solo para la atención maternoinfantil sino para cualquiera de las especialidades médicas.

Este hecho, sumado al permanente déficit presupuestal está gravitando sobre las causais de muertes maternas.

La infección sigue ocupando el primer 
lugar y es el aborto con sus complicaciones el primer desencadenante. En nuestro medio Márquez (5), reveló el mismo resultado. Hasta cuándo, nos preguntamos nosotros, vamps a seguir imputando al aborto este porcentaje? Cuando en los países desarrollados se ha logrado disminuír el número de muertes por pre-eclampsia, entre nosotros no se ha observado esta modificación, Tomkinson (6) informa que en Inglaterra y Gales la pre-eclampsia fué la causa de muerte en 246 pacientes durante el año de 1969.

La tasa global de mortalidad materna fué de 25.5 por 10.000 nacidos vivos. Esta cifra contrasta con la dada por el mismo autor (6) de 5.4 por 10.000 nacidos vivos en 1952 y de 1.5 en 1969.

Nuestra tasa es más del doble de la encontrada en el Hospital de Barquisimeto (Venezuela) (7).

Sólo el $11.4 \%$ de las pacientes fallecidas asistió a la consulta prenatal; si aceptamos que la asistencia antes del parto es importante para prevenir la pre-eclampsia y la eclampsia, este factor está repercutiendo en dichas muertes.

Se considera que a medida que se incrementa la consulta prenatal la tasa de mortalidad materna debe descender.

El número de nacidos vivos fué de 47 (31.5\%), éste dato puede ser el reflejo de la poca importancia que en ocasiones se brinda al feto cuando la madre está en peligro de muerte. En otras ocasiones se debe a la demora para consultar.

En todo centro hospitalario debe existir un registro y un comité de muertes maternas y se debe velar por obtener el estudio anatomopatológico completo (autopsia) a todas las pacientes fallecidas.

Mientras en otros hospitales es del 49 al $79.1 \%$ (8) (9), entre nosotros sólo alcanza al $41.6 \%$ el número de autopsias realizadas.

\section{RESUMEN :}

Se analiza 149 historias, de madres que murieron en el Hospital Universitario San Vicente de Paúl en el lapso comprendido entre 1963 y 1976 inclusive.

Se estudiaron las siguientes variables:
1. Edad de la matire

2. Procedencia

3. Estado civil

4. Semanas de gestación

5. Paridad

6. Atención prenatal

7. Causa de la muerte

8. Estado final del recién nacido

Cuando la muerte se debió al aborto se buscó el grado de infección y la iniciación del mismo (espontáneo o provocado).

Las causas de muerte se dividieron en: Directas, indirectas y no relacionadas. Además en muertes por aborto, parto y puerperio.

La tasa de mortalidad materna fué de 25.5 por 10.000 nacidos vivos.

Las siguientes fueron las causas de muerte:

1. Infección $=44.3 \%$

2. Indirectas $=21.4 \%$

3. Toxemia $=20.1 \%$

4. Hemorragia $=14.2 \%$

Cincuenta y cuatro pacientes murieron como consecuencia del aborto, esto representa el $36.2 \%$ de todas las muertes. Las restantes, fueron por complicaciones del parto o del puerperio.

De las 66 madres muertas por infección el $57.5 \%$ se debió a un aborto infectado y el $70 \%$ de las que fallecieron por aborto tuvieron como complicación una infección.

El $63.6 \%$ de las muertes debidas a hemorragias presentaron placenta previa, desprendimiento prematuro de la misma o ruptura uterina. De las 29 muertes debidas a la toxemia el $24.1 \%$ correspondió a la eclampsia.

El estudio anatomopatológıco fué practicado en el $41.6 \%$ de las madres fallecidas.

La consulta prenatal sólo fué hecha en 17 pacientes $(11.4 \%)$

\section{CONCLUSIONES:}

7.1. La tasa de mortalidad materna en el Hospital Universitario San Vicente de Paul fué de 25.5 por 10.000 nacidos vivos.

7.2 La infección fué la principal causa de 
muerte con $44.3 \%$

7.3. El $36.2 \%$ de las pacientes fallecidas presentaron un aborto

$7.4 \mathrm{El} 57.5 \%$ de las madres muertas por infección presentaron un aborto séptico.

7.5 El $20.1 \%$ fallecieron por una pre-eclampsia o eclampsia.

7.6 Sólo el $11.4 \%$ de las pacientes asistió a la consulta prenatal.

7.7. La autopsia se practicó en el $41.6 \%$ de las muertes.

7.8. Es indispensable el estudio piramidal y continuado de las muertes maternas en los hospitales universitarios, que con criterios uniformes pueda ser utilizado para la enseñanza de atención maternoinfantil.

\section{SUMARY}

An analysis was made of the 149 cases of mothers who had died at the Hospital Universitario San Vicente de Paul between 1963 and 1973.

The following variables were studied:

1 Age of the mother

2 Place of origin

3 Marital status

4 Weeks og gestation

5 Childbirth

6 Prenatal care

7 Cause of death

8 Final state of newborn

When death was caused by an abortion, the degree of infection and the type of abortion (whether induced or spontaneous) were established.

The causes of deaths were divided into: direct, indirect, and non-related; as well as well as abortion, delivery, and puerperium.

The maternal death rate was 25.5 per 10.0 00 births.

The following were the causes of death:

$\begin{array}{lll}1 & \text { Infection } & 44.3 \% \\ 2 & \text { Indirect } & 21.4 \% \\ 3 & \text { Toxemia } & 20.1 \% \\ 4 & \text { Hemorrhage } & 14.2 \%\end{array}$

$36.2 \%$ (54) of all deaths were caused by abortions, and the remaining deaths were due to delivery problems or pauperium.

$57.5 \%$ (66) of all deaths caused by infection were due to an infected abortion, and $70 \%$ of those who died as a result of an abortion, showed complications consisting in infections. $63.6 \%$

$63.6 \%$ of the deaths caused by hemorrhages showed signs of previous placenta, premature detachment or rupture of the uterine. Of the 29 deaths caused by toxemia, $24.1 \%$ resulted from eclampsia.

An anatomopathological study was carried out on $41.6 \%$ of the deceased mothers.

Prenatal consultations were made in only $11.4 \%$ (17) of the casses.

No maternal deaths occurred in the group that was examined.

The G.I. was $6 / 11$ in one patient whose condition worsened as a result of subcapsular hematomas of the liver. Thirty fetal deaths occurred: The G.I. decreased in 25 cases and remained equal in 5 . In 2 cases wrere the G.I. has risen, the fetus was born alive.

It was concluded that the G.I. has no practical value in the prediction of the mater nal or fetal future.

\section{BIBLIOGRAFIA:}

(1) Williams, C.D. and Jelliffe, D.B. "Mother and Child Health Delivering The Services" Oxford University Press Toronto 1972.

(2) Rice Puffer, R. and Wynne Griffth, G. "Características de la mortalidad urbana" Informe de La Investigación Interamericana de Mortalidad Publicación No. 151 O.M.S. Pág. 191 Mayo 1968.

(3) Committee on Maternal and Child Care of the A.M.A. "A guide for maternal death studies"' 1964.

(4) Jubiz H., A. y Sánchez M., C. "Mortalidad materna comparativa entre dos períodos 1963 - 1967 y 1968 - 1972', Rev. Col. Obst. Ginec. 27: (3) 129-136 1976.

(5) Márquez, L. "Mortalidad Materna en el Hospital Universitarios San Vicente de Paúl"' Mimeografiado 1962.

(6) Tomkinson, J.S. "Mortalidad Materna en Inglaterra”' Mimeografiado 1970.

(7) Crespo, J. "Mortalidad Materna en el Hospital Antonio María Pineda" Barquisimeto - Venezuela 1959 a 1968. Rev. Gin. Obst. de Ven. 30: 497 - 504. 1970.

(8) Gibbs, C. E. and Locke, W. "Maternal deaths in Texas 1969 to 1973 " Am. J. Obstet. Gynecol. 126: 687. 1976.

(9) Hughes, E.; Cochrane, N. "Maternal Mortality Study 1970 - 1975" Obstet. Gynec. Survey 32: 371. 1977. 